\title{
ON SOME EXAMPLES OF GROUP ACTIONS AND GROUP EXTENSIONS
}

\author{
Alejandro Adem* and Ergün Yalçın \\ Department of Mathematics \\ University of Wisconsin \\ Madison, WI 53706 USA
}

\section{$\S 0$ Introduction}

It is well-known that finite groups which can act freely on a sphere must be rather special. To be precise we know that their abelian subgroups are cyclic (see [CE], Theorem XII.11.6) and conversely that any group satisfying this condition acts freely on a finite complex with the homotopy type of a sphere (a result due to Swan $[\mathrm{Sw}]$ ). How does this extend to other groups? The first fact to keep in mind is that any finite group will act freely on some product of equidimensional spheres (see $[\mathrm{O}]$ ). Hence it makes sense to determine the minimal number $n$ such that $G$ acts freely on a finite complex $X \simeq\left(\mathbb{S}^{m}\right)^{n}$ for some $m$. In this paper we address this problem for finite 2-groups; in this context the following interesting question has been raised (see [ACPW], Benson's problem list):

Question: If $G$ is a finite 2-group, is $n$ equal to the rank of its largest elementary abelian subgroup?

Although there exist examples of groups of composite order for which the answer is negative (the alternating group $\mathcal{A}_{4}$ is such an example, see [O]), the methods used do not apply to the case of 2-groups, and this problem remains open even in the rank two situation.

In this note we present an analysis of this problem for certain classes of 2-groups, and show that it relates to interesting questions about group extensions. We first consider 2 -groups $G$ such that every element of order 2 is central (the $2 \mathrm{C}$ condition). If such a group acts freely on a product $X \simeq\left(\mathbb{S}^{m}\right)^{n}$ we show that the action is homologically trivial mod 2 and that the mod 2 cohomology of the orbit space can be expressed as $H^{*}\left(X / G, \mathbb{F}_{2}\right) \cong H^{*}\left(G, \mathbb{F}_{2}\right) /\left(\mu_{1}, \ldots, \mu_{n}\right)$ where $\mu_{1}, \ldots, \mu_{n} \in H^{m+1}\left(G, \mathbb{F}_{2}\right)$ form a regular sequence of maximal length. After noting that such an action can always be constructed using representation theory, we apply this to provide a direct geometric proof of a recent cohomological characterization of groups with the $2 \mathrm{C}$ property (see $[\mathrm{AK}]$ ).

Next we consider actions of groups expressed as an extension

$$
1 \rightarrow V \rightarrow G \rightarrow W \rightarrow 1
$$

\footnotetext{
* Partially supported by an NSF grant, the MPIM-Bonn, Université de Paris 7 and CRM-Barcelona.
} 
where $V$ and $W$ are elementary abelian 2-groups and $V$ is of maximal rank. Using cohomological arguments we find a bound on the rank of $W$; in particular if the induced action of $W$ on $V$ permutes a basis, then we obtain

$$
\operatorname{dim} W \leq \operatorname{dim} V+\operatorname{dim} V^{W}
$$

These methods are then used to show that if $W=(\mathbb{Z} / 2)^{r}$ acts freely on a finite complex $X$ with cohomology generated by one-dimensional classes and such that the action permutes a basis for $H_{1}\left(X, \mathbb{F}_{2}\right)$, then

$$
r \leq \operatorname{dim} H_{1}\left(X, \mathbb{F}_{2}\right)+\operatorname{dim} H_{1}\left(X, \mathbb{F}_{2}\right)^{W} \leq 2 \operatorname{dim} H_{1}\left(X, \mathbb{F}_{2}\right) .
$$

From this we derive another

Question: If $W=(\mathbb{Z} / 2)^{r}$ acts freely on a finite complex $X$ with cohomology generated by one dimensional classes, does it follow that $r \leq 2 \operatorname{dim} H_{1}\left(X, \mathbb{F}_{2}\right)$ ?

Using geometric restrictions, we show that if a group $G$ defined as in (E) acts freely on $X \simeq\left(\mathbb{S}^{m}\right)^{n}$, where $n$ is the rank of $V$ and such that $r k W \geq 2 n+1$, then the orbit space is not homotopy equivalent to $\left(\mathbb{R} P^{m}\right)^{n}$; indeed this indicates that 'exotic' intermediate orbit spaces must arise for free actions of group extensions such as (E) where $|W|$ is large relative to $|V|$. However, for $\left(\mathbb{R} P^{m}\right)^{n}$ we can show that if $(\mathbb{Z} / 2)^{r}$ acts freely on it, then $r \leq 2 n$.

By using extensions of type (E) having $W$ of very large rank (introduced by Ol'shanskii $[\mathrm{Ol}]$ ), we construct some striking examples of very large elementary abelian 2-groups acting on products of projective spaces with relatively small isotropy. For example, we show that there is an action of $(\mathbb{Z} / 2)^{1249}$ on $X=\left(\mathbb{R} P^{2^{1298}-1}\right)^{50}$ with isotropy subgroups of rank at most 50. Finally we use these groups to prove the following

\section{Proposition}

At least one of the two questions above has a negative answer.

Unless stated otherwise, all coefficients will be taken in $\mathbb{F}_{2}$, the field with two elements, so they are suppressed throughout. In addition we will assume that the dimensions of the spheres appearing in our arguments are larger than 7 . We are grateful to Jon Alperin and George Glauberman for pointing out Ol'shanskii's examples to us, to Dave Benson for his very helpful comments and to the referee for his valuable advice.

\section{$\S 1$ Free Group Actions and Orbit Spaces}

Let $X$ denote a finite complex with a free action of a finite group $G$. To compute the cohomology of the orbit space $X / G$ there is a spectral sequence (Cartan-Leray) of the form $E_{2}^{p, q}=H^{p}\left(G, H^{q}(X)\right)$ converging to $H^{p+q}(X / G)$. In this note we will be interested in 2-group actions on products of equidimensional spheres (or on finite complexes homotopy equivalent to them).

To begin we recall a result due to $\mathrm{G}$. Carlsson $[\mathrm{C}]$ for actions of $G=(\mathbb{Z} / 2)^{n}$ :

\section{Proposition 1.1}

If $G=(\mathbb{Z} / 2)^{n}$ acts freely on $X \simeq\left(\mathbb{S}^{m}\right)^{n}$, trivially in homology, then there exist classes $\mu_{1}, \ldots, \mu_{n} \in H^{m+1}(G)$ such that $H^{*}(X / G) \cong H^{*}(G) /\left(\mu_{1}, \ldots, \mu_{n}\right)$. 
The proof requires showing that we may take the $\mu_{i}$ to be transgressions of the cohomology generators for the product of spheres and that these classes form a regular sequence $\mu_{1}, \ldots, \mu_{n}$ in $H^{*}(G)$. More generally given a homologically trivial free action of any finite group on a product of equidimensional spheres, the cohomology of the orbit space can be expressed as a quotient of the type in 1.1 if the transgressions of the generators on the fiber form a regular sequence.

Now by the results in $[\mathrm{AB} 2]$, we know that any free action of $(\mathbb{Z} / 2)^{n}$ on $\left(\mathbb{S}^{m}\right)^{n}$ is homologically trivial if $m>7$ (our standing assumption). With this additional information we can extend 1.1 to a more general class of groups.

\section{Theorem 1.2}

Let $G$ denote a finite 2 -group such that every element of order 2 in $G$ is central. If $G$ acts freely on $X \simeq\left(\mathbb{S}^{m}\right)^{n}$, where $n$ is the rank of the largest elementary abelian 2-subgroup in $G$, then

(1) $G$ acts homologically trivially on $X$ and

(2) the transgressions of the cohomology generators for the spheres form a regular sequence $\zeta_{1}, \ldots, \zeta_{n} \in H^{m+1}(G)$ and so

$$
H^{*}(G) /\left(\zeta_{1}, \ldots, \zeta_{n}\right) \cong H^{*}(X / G)
$$

Proof: Let us first assume homological triviality. We will use the fact that a group as above has Cohen-Macaulay cohomology (a result due to Duflot [D]). It suffices to show that $H^{*}(G)$ is finitely generated as a module over the subalgebra generated by the $\zeta_{i}$. However note that there is a unique maximal elementary abelian subgroup $E \subset G$ which has rank $n$ and is of course central. By Quillen's $F$-isomorphism theorem, it suffices to show that $\operatorname{res}_{E}^{G}\left(\zeta_{1}\right), \ldots, \operatorname{res}_{E}^{G}\left(\zeta_{n}\right)$ form a regular sequence in $H^{*}(E)$. This fact follows from 1.1 .

To prove homological triviality, we consider the exact sequence

$$
H^{m}(X / E) \longrightarrow H^{m}(X) \stackrel{d_{m+1}}{\longrightarrow} H^{m+1}(E)
$$

which arises from the spectral sequence associated to the action of $E$. Note that by our hypotheses the $E$ action is homologically trivial. As $G$ centralizes $E, G / E$ will act on this sequence, trivially on $H^{*}(E)$. Now $d_{m+1}$ has rank $n$, hence it is injective and so we deduce that the action must be trivial on the entire cohomology, completing the proof.

More generally what we have is that if a 2-group of rank $n$ acts freely on a product of $n$ equidimensional spheres, then the centralizers of the elementary abelian subgroups of maximal rank fulfill the conditions above. In particular if $H^{*}(G)$ is Cohen-Macaulay, then the associated transgressions $\mu_{1}, \ldots, \mu_{n} \in H^{m+1}(G)$ will be a regular sequence (note that in addition the centralizers mentioned before will detect the cohomology). Hence a lower bound on $m+1$ is given by the minimal dimension which allows for a collection of equidimensional regular elements which has the largest length.

Note that for an arbitrary finite group satisfying the $2 \mathrm{C}$ condition it would suffice to require that the $G$ action have odd order isotropy subgroups to obtain a version of 1.2. Also we should mention that Duflot's result is proved using a particular action of $G$ on 
a product of spheres. The following construction provides one. Let $x_{1}, \ldots, x_{n}$ be a basis for $E$, the unique maximal elementary abelian subgroup in $G$, a group satisfying the $2 \mathrm{C}$ condition. Let $\chi_{i}$ denote a non-trivial 1-dimensional real representation for $C_{i}=<x_{i}>$; denote $V_{i}=\operatorname{Ind}_{C_{i}}^{G}\left(\chi_{i}\right)$. Let $G$ act diagonally on $X=S\left(V_{1}\right) \times \ldots \times S\left(V_{n}\right) \cong\left(\mathbb{S}^{|G| / 2-1}\right)^{n}$; then one can easily verify that the action restricted to the central subgroup $E \subset G$ is free. As $E$ contains every involution in $G$, all the isotropy subgroups must be of odd order.

More generally if $V \cong(\mathbb{Z} / 2)^{k}$ is a central subgroup in $G$ the above construction can be used to provide an action of $G$ on $X=\left(\mathbb{S}^{|G| / 2-1}\right)^{k}$ such that if $\zeta_{1}, \ldots, \zeta_{k}$ are the associated transgressions, then they form a regular sequence and

$$
H^{*}(G) /\left(\zeta_{1}, \ldots, \zeta_{k}\right) \cong H_{G}^{*}(X)
$$

where $H_{G}^{*}(X)$ denotes the equivariant cohomology of $X$. If $k$ is equal to the rank of $G$ at $p=2$, we recover the 2 -central case above.

The structural results above can be used to construct some very special cohomology classes for finite 2-groups. If $G$ is a 2-group such that every element of order 2 is central, then we may write it as $G \cong G_{0} \times(\mathbb{Z} / 2)^{n}$, where $G_{0}$ is a group (also satisfying the 2-central condition) such that every maximal subgroup has the same rank as $G_{0}$ itself.

\section{Proposition 1.3}

Suppose that a group $G_{0}$ as above acts freely on $X=\left(\mathbb{S}^{m}\right)^{n}$, where $n$ is the rank of $G_{0}$. Let $\pi_{G_{0}}: X / G_{0} \rightarrow B G_{0}$ denote the usual classifying map. If $x \in H^{m n}\left(G_{0}\right)$ is such that $\pi_{G_{0}}^{*}(x) \neq 0$, then there exists a non-zero class $w \in H^{m n}\left(G_{0}\right)$ such that $\operatorname{res}_{H}^{G_{0}}(w)=0$ for all proper subgroups $H \subset G_{0}$.

Proof: To prove this we take $H \subset G_{0}$ a maximal subgroup and consider the following commutative diagram

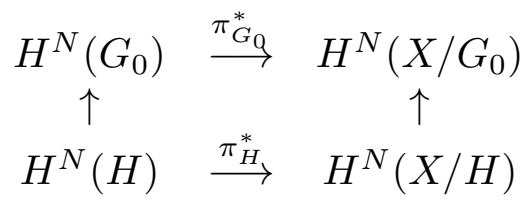

where the two vertical arrows are transfer maps and $N=m n$. Note that as $H$ still has maximal rank, our previous arguments show that $\pi_{H}^{*}$ is onto. Furthermore the right hand vertical map is surjective, as its image is the kernel of multiplication by the one dimensional class corresponding to $H$; as $N$ is the top dimension this is the zero map. If we take the class $x \in H^{N}\left(G_{0}\right)$, then there exists a $y \in H^{N}(H)$ such that $t r \cdot \pi_{H}^{*}(y)=\pi_{G_{0}}^{*}(x)$; hence if we take $z=\operatorname{tr}(y)$, then $z$ is a class which satisfies the same hypothesis as $x$, but is a transfer image. Continuing like this, using index 2 subgroups, we can construct a class $w \in H^{N}\left(G_{0}\right)$ which is in the image of the transfer from $H^{*}(E)$ (E the unique maximal elementary abelian subgroup) which still satisfies the same condition that $x$ does.

Now let $K$ denote any maximal subgroup, we will use the double coset formula to compute $\operatorname{res}_{K}^{G_{0}}(w)$. If $w=\operatorname{tr}_{E}^{G_{0}}(u)$, then the following terms will appear in this formula: $t r_{K \cap E^{g}}^{K} \cdot r e s_{E^{g} \cap K}^{E^{g}}\left(c_{g} u\right)$, where $c_{g}$ is the map induced by conjugation by $g$ and the summation is taken over $\bar{g} \in K \backslash G_{0} / E$. In our case $E$ is central, hence the conjugation action is trivial, $E=E^{g}, E^{g} \cap K=E$ and $K \backslash G_{0} / E$ has $\left[G_{0}: K\right]$ distinct elements. Hence we obtain

$$
\operatorname{res}_{K}^{G_{0}}(w)=\left[G_{0}: K\right] u=0
$$


and the proof is complete.

Note that for the case of a general $G \cong G_{0} \times(\mathbb{Z} / 2)^{n}$, we can take $\gamma$ to be the product of all one dimensional classes in $H^{*}\left(G_{0} \times(\mathbb{Z} / 2)^{n}\right)$ which involve non-zero terms from the elementary abelian factor, and if $w$ is chosen as above, then $w \gamma$ will be a non-zero class restricting trivially on all proper subgroups in $G$. A more complicated but purely algebraic construction of such classes was provided in $[\mathrm{AK}]$. From this it can be shown that the finite groups which have Cohen-Macaulay mod 2 cohomology with undetectable elements are precisely the 2-groups such that every element of order two is central.

\section{$\S 2$ Group Extensions}

In this section we will consider actions of 2-groups which can be described as extensions of the form

$$
1 \rightarrow V \rightarrow G \rightarrow W \rightarrow 1
$$

where $V$ and $W$ are both elementary abelian of finite rank. In addition we will assume that $V$ is of maximal rank in $G$. We begin with a purely group-theoretic fact.

\section{Proposition 2.1}

Let $G$ be an extension as above, where $W$ acts on a basis for $V$ via permutations. Then $\operatorname{dim} W \leq \operatorname{dim} V+\operatorname{dim} V^{W}$.

Proof: Choose $H \subset W$ the subgroup of elements which act trivially on $V$ and consider the five-term exact sequence associated to the extension $1 \rightarrow V \rightarrow \tilde{H} \rightarrow H \rightarrow 1$ :

$$
0 \rightarrow H^{1}(H) \rightarrow H^{1}(\tilde{H}) \rightarrow H^{1}(V) \stackrel{d_{2}}{\rightarrow} H^{2}(H) \rightarrow H^{2}(\tilde{H}) .
$$

The quotient group $W / H$ acts on this, trivially on $H^{2}(H)$, hence we obtain that the coinvariants $H^{1}(V)_{W}$ have rank at least as large as the dimension of $i m d_{2}$. We claim that $i m d_{2}$ has rank at least $r k(H) / 2$. Suppose otherwise, then the $\mathrm{k}$-invariants of the extension would form a collection of say $q$ quadratic polynomials in $H^{*}(H)$, a polynomial ring on at least $2 q+1$ variables. This implies that they have a common zero (see [G], pages 11 and 18); therefore an extra $\mathbb{Z} / 2$ summand would split off and hence $\tilde{H}$ would have rank larger than that of $V$, which is impossible. Hence by dualizing we conclude that $r k H \leq 2 \operatorname{dim} V^{W}$. Now $W / H$ acts faithfully on $V$, a permutation module and so one can easily show that $r k W / H \leq \operatorname{dim} V-\operatorname{dim} V^{W}$ from which we can then infer that

$$
r k W \leq \operatorname{dim} V+\operatorname{dim} V^{W}
$$

\section{Corollary 2.2}

If all elements of order 2 in $G$ are central then $r k G / V \leq 2 r k V$.

More generally note that if $Q \subset G L(V)$ is elementary abelian, then $r k Q \leq(r k V)^{2} / 4$, and so the proof above can be modified to yield the quadratic bound

$$
\operatorname{dim} W \leq 2 \operatorname{dim} V^{W}+(\operatorname{dim} V)^{2} / 4
$$


We now switch to topology to prove a result analogous to 2.1 for group actions.

\section{Theorem 2.3}

Let $X$ denote a finite complex such that $H^{*}(X)$ is generated by one dimensional classes. If $W=(\mathbb{Z} / 2)^{r}$ acts freely on $X$ inducing a permutation module on $H_{1}(X)$, then

$$
r \leq \operatorname{dim} H_{1}(X)+\operatorname{dim} H_{1}(X)^{W} \leq 2 \operatorname{dim} H_{1}(X) .
$$

Proof: As before we choose $H \subset W$, the subgroup of elements acting trivially on $H^{1}(X)$, and hence trivially on all the cohomology. Consider the transgression map $d_{2}: H^{1}(X) \rightarrow$ $H^{2}(H)$ arising from the spectral sequence for the action of $H$ (as discussed in $\S 1$ ). Now we claim as before that $i m d_{2}$ has dimension at least $r k(H) / 2$. Suppose otherwise, then we can find a cyclic subgroup $C \subset H$ such that $\operatorname{res}_{C}^{H} \cdot d_{2}=0$, and hence the corresponding differential $d_{2}$ will be identically zero in the spectral sequence for the action of $C$. As $H^{*}(X)$ is generated by one dimensional classes, this means that in fact the spectral sequence collapses, and so the $E_{\infty}$ term is infinite dimensional. However the spectral sequence converges to the cohomology of the finite complex $X / C$, whence we obtain a contradiction.

The rest of the proof now follows directly from the arguments used in proving 2.1.

From the topological point of view, the following question seems very natural:

\section{Question A}

If $W=(\mathbb{Z} / 2)^{r}$ acts freely on a finite complex $X$ with cohomology generated by one dimensional classes, does it follow that $r \leq 2 \operatorname{dim} H_{1}(X)$ ?

Now we consider actions on products of spheres by group extensions of the type defined in this section. As before let us assume that $V$ has maximal rank $n$, and suppose that $G$ acts freely on $X \simeq\left(\mathbb{S}^{m}\right)^{n}$. As we have seen, $V$ acts homologically trivially on $H^{*}(X)$. More generally, we know by a Hopf invariant argument (see [AB2]) that $G$ acts on $H^{m}(X)$ by permuting the canonical basis. Hence we have a fundamental exact sequence of $G / V=W$ modules:

$$
0 \rightarrow H^{m}(X) \stackrel{d_{m+1}}{\longrightarrow} H^{m+1}(V) \longrightarrow H^{m+1}(X / V) \rightarrow 0
$$

where the module on the left is a permutation module. Or equivalently, $W$ permutes the $\mathrm{k}$-invariants $\mu_{1}, \ldots, \mu_{n}$, where

$$
H^{*}(X / V) \cong \mathbb{F}_{2}\left[x_{1}, \ldots, x_{n}\right] /\left(\mu_{1}, \ldots, \mu_{n}\right)
$$

This provides a geometric constraint on the possible group extension. An immediate consequence is the following.

\section{Theorem 2.4}

$$
\text { If } X / V \simeq\left(\mathbb{R} P^{m}\right)^{n} \text {, then } r k W \leq r k V^{W}+r k V \text {. }
$$

Proof: Our hypotheses imply that

$$
H^{*}(X / V) \cong \mathbb{F}_{2}\left[y_{1}, \ldots, y_{n}\right] /\left(y_{1}^{m+1}, \ldots, y_{n}^{m+1}\right)
$$


for one dimensional classes $y_{1}, \ldots, y_{n}$. If we consider these k-invariants as elements in $H^{*}(V)$, then they must span a $W$-submodule $M$. Write $m+1=2^{r} k$, where $k$ is an odd number. If $k>1$, then by the binomial coefficient theorem it is easy to see that the vectors $y_{1}, \ldots, y_{n}$ must be permuted by the action of $W$, as otherwise their $m+1$ powers would not span a $W$-submodule (note that this argument does not require any homotopy theory). If $k=1$ and so $m+1$ is a power of two, then the iterated squaring map induces a $W$ isomorphism between the permutation module $M$ and $H^{1}(X / V)$. Applying 2.3 completes the proof.

\section{Corollary 2.5}

If $W=(\mathbb{Z} / 2)^{r}$ acts freely on $Y \simeq\left(\mathbb{R} P^{m}\right)^{n}$, then $r \leq 2 n$.

If $m=4 k+1$, the proof can be modified to show that in fact $r \leq n$. The point is that in this case the ideal generated by the transgressions is invariant under the action of the Steenrod algebra. A result due to Carlsson [C] implies that if there were more than $n$ variables, then the quadratic polynomials would have a common zero. This observation is due to Cusick $[\mathrm{Cu}]$. Recall (see $[\mathrm{AB} 1]$ ) that the free 2-rank of symmetry of a finite complex $X$ is the rank of the largest elementary abelian 2-group which acts freely on it. Our results extend those of Cusick $[\mathrm{Cu}]$ to actions which are not homologically trivial, and can be summarized in the following

\section{Theorem 2.6}

If $X=\left(\mathbb{R} P^{m}\right)^{n}$ and $\phi(X)$ denotes its free 2-rank of symmetry, then we have

$$
\phi(X)= \begin{cases}0 & \text { if } m \equiv 0,2 \bmod 4 \\ n & \text { if } m \equiv 1 \bmod 4 \\ 2 n & \text { if } m \equiv 3 \bmod 4\end{cases}
$$

Proof: We have already established the upper bounds for $m$ odd. For $m$ even we have $\chi(X)=1$, whence no non-trivial finite group can act freely on $X$. To complete the proof it suffices to to observe that the standard free actions of $\mathbb{Z} / 4$ on $\mathbb{S}^{4 q+1}$ and of the quaternion group $Q_{8}$ on $\mathbb{S}^{4 q+3}$ give rise to actions on real projective space whose products achieve the desired upper bounds.

\section{Corollary 2.7}

An extension $G$ of type (2.0) acts freely and homologically trivially on $X \simeq\left(\mathbb{S}^{m}\right)^{n}$ such that $X / V \simeq\left(\mathbb{R} P^{m}\right)^{n}$ if and only if every element of order 2 in $G$ is central.

Proof: We have already seen that any 2-group satisfying the $2 \mathrm{C}$ condition acts freely and homologically trivially on a product of equidimensional spheres such that the generators of the maximal (central) 2-torus act via multiplication by -1 on the factors. Hence $X / V \cong$ $\left(\mathbb{R} P^{m}\right)^{n}$ in this case. Conversely the hypotheses imply that the $W$ action on $H^{1}(V)$ is trivial, hence $V$ is central and of maximal rank, which implies the $2 \mathrm{C}$ condition.

We now exhibit some interesting examples of group extensions.

\section{Examples 2.8}

The following examples appear in [Ol]. Let $\Phi=\left\{\phi_{1}, \ldots, \phi_{t}\right\}$ denote a collection of skew-symmetric bilinear forms on an $n$-dimensional $\mathbb{F}_{2}$-vector space $M$. A group $G_{\Phi}$ can be defined as follows. As generators we will take elements 


$$
a_{1}, \ldots, a_{n}, \quad b_{1}, \ldots, b_{t}
$$

subject to the relations

$$
a_{i}^{2}=b_{j}^{2}=\left[a_{i}, b_{j}\right]=\left[b_{s}, b_{u}\right]=1
$$

and

$$
\left[a_{i}, a_{j}\right]=b_{1}^{\phi_{1}\left(a_{i}, a_{j}\right)} \ldots b_{t}^{\phi_{t}\left(a_{i}, a_{j}\right)}
$$

for $i, j=1,2, \ldots, n$ and $s, u=1, \ldots, t . G_{\Phi}$ is a class 2 nilpotent group, $B=<b_{1}, \ldots, b_{t}>$ is central and $G_{\Phi} / B \cong M=<a_{1} B, \ldots, a_{n} B>$.

In [Ol] it is shown that given an integer $k>0$ such that $n<t(k-1) / 2$, then we may choose $\phi_{1}, \ldots, \phi_{t}$ such that the elementary abelian subgroups in the associated group $G_{\Phi}$ are of rank at most $t+k-1$.

For example, if $k=51, t=50$ and $n=1249$, then $G_{\Phi}$ fits into an extension of the form

$$
1 \rightarrow(\mathbb{Z} / 2)^{T} \rightarrow G_{\Phi} \rightarrow(\mathbb{Z} / 2)^{N} \rightarrow 1
$$

where $T \leq 100, N \geq 1199$ and $T$ is the rank of $G_{\Phi}$.

Using the construction described in $\S 1$, we obtain an action of $G_{\Phi}$ on $X=\left(\mathbb{S}^{m}\right)^{50}$, $m=2^{1298}-1$, such that the generators of $B$ act via multiplication by -1 on the spheres and of course the maximal rank of an isotropy subgroup is $T-50 \leq 50$. Dividing out by the $B$ action we obtain an action of $(\mathbb{Z} / 2)^{1249}$ on $\left(\mathbb{R} P^{m}\right)^{50}$ with isotropy of rank at most 50 .

To tie things together we now introduce a well-known question on group actions (see [ACPW], D. Benson's problem list).

\section{Question B}

If $G$ is any finite 2-group of rank $l$, does there exist a finite complex $X \simeq\left(\mathbb{S}^{m}\right)^{l}$ such that $G$ acts freely on $X$ ?

\section{Proposition 2.9}

Questions $A$ and $B$ cannot both have affirmative answers.

Proof: Suppose that question B were true and take $G_{\Phi}$ as before. Then we would have a free action of $(\mathbb{Z} / 2)^{N}$ on $X /(\mathbb{Z} / 2)^{T}$, where $X \simeq\left(\mathbb{S}^{m}\right)^{T}$. As $T$ is the rank of the group, a maximal elementary abelian subgroup $V$ acts homologically trivially on $X$, and as we have seen

$$
H^{*}(X / V) \cong \mathbb{F}_{2}\left[x_{1}, \ldots, x_{T}\right] /\left(\mu_{1}, \ldots, \mu_{T}\right)
$$

where the $x_{i}$ are one dimensional and the $\mu_{j}$ form a regular sequence in the polynomial algebra. Note however that $N \geq 1199$, whereas $T \leq 100$ ! This negates question A; indeed $G_{\Phi}$ must provide a counterexample to one of the two questions. Hence a proof of one of them will disprove the other one.

A concrete result which we do have is the following. 


\section{Theorem 2.10}

If $G$ as in (2.0) acts freely on $X \simeq\left(\mathbb{S}^{m}\right)^{n}$, where $n=r k V$ and $r k W \geq 2 n+1$, then $X / V$ is not homotopy equivalent to $\left(\mathbb{R} P^{m}\right)^{n}$.

It should be noted that the dimension of the spheres involved in our free actions must be sufficiently large to produce enough homology. More precisely, suppose we know that an extension $G$ as in (2.0) acts freely on $X \simeq\left(\mathbb{S}^{m}\right)^{t}$, where we assume $\operatorname{dim} V=$ $r k G=t$. As we know, the cohomology of the orbit space $X / V$ will be of the form $\mathbb{F}_{2}\left[x_{1}, \ldots, x_{t}\right] /\left(\mu_{1}, \ldots, \mu_{t}\right)$; note that the total dimension of the cohomology is $(m+1)^{t}$. Using cohomological exponents it is possible to show that

$$
\operatorname{dim} G / V \leq(m+1) t
$$

We sketch the proof: there is an index $2^{t}$ subgroup $H \subset G$ which acts trivially on $H^{*}(X, \mathbb{Z})$. Hence $H / H \cap V \subset G / V$ acts freely and preserving orientation on $X / H \cap V$. Moreover it acts trivially on the torsion free part of $H^{*}(X / H \cap V)$. Decomposing the top class in terms of $m$ dimensional classes and using a theorem of Browder [B], we can obtain $\log _{2}|H / H \cap V| \leq \operatorname{dim} X / H \cap V$, and so we conclude $\operatorname{dim} G / V-t \leq \operatorname{dim} H / H \cap V \leq m t$, whence the result follows.

A conjecture due to Carlsson (see [AB1]) would imply that in all cases

$$
2^{\operatorname{dim} G / V} \leq(m+1)^{t}
$$

For example if we take $G_{\Phi}$ as before, then the first inequality yields $1099 \leq 100 \mathrm{~m}$, whence $m \geq 11$. The conjectured inequality would yield $2^{1199} \leq(m+1)^{100}$ from which we would deduce $m \geq 2^{11}-1$.

\section{REFERENCES}

[AB1] Adem, A. and Browder, W., "The Free Rank of Symmetry of $\left(\mathbb{S}^{n}\right)^{k}$," Inventiones Math. 92 (1988), 431-440.

[AB2] Adem, A. and Benson, D., "Elementary Abelian Groups Acting on Products of Spheres," Math. Zeitschrift, to appear.

[ACPW] Adem, A., Carlson, J., Priddy, S., Webb, P., (editors) Proceedings of Symposia in Pure Mathematics, Seattle 1996 AMS Summer Institute Volume (1997).

[AK] Adem, A. and Karagueuzian, D., "Essential Cohomology of Finite Groups," Comm. Math. Helvetici 72 (1997), 101-109.

[B] Browder, W., "Cohomology and Group Actions," Inventiones Math. 71 (1983), 599607.

[C] Carlsson, G., "On the Nonexistence of Free Actions of Elementary Abelian Groups on Products of Spheres," Am. J. Math. 102 (1980), 1147-1157.

[CE] Cartan, H. and Eilenberg, S., Homological Algebra, Princeton University Press (1956).

[Cu] Cusick, L.. "Elementary Abelian 2-Groups that Act Freely on Products of Real Projective Spaces," Proc. Am. Math. Soc. Vol.87, No.4 (1983), 728-730. 
[D] Duflot, J., "Depth and Equivariant Cohomology," Comm. Math. Helv. 56 (1981), 627-637.

[G] Greenberg, M., Lectures on Forms in Many Variables, Benjamin, New York 1969.

[O] Oliver, B., "Free Compact Group Actions on Products of Spheres," in Springer-Verlag Lecture Notes in Mathematics, Vol. 763 (Arhus 1978 Algebraic Topology Volume), pp. 539-548.

[Ol] Ol'shanskii, A., "The Number of Generators and Orders of Abelian Subgroups of Finite $p$-groups," Math. Notes Vol. 23. No. 3 (1978), 183-185.

[Sw] Swan, R. G., "Periodic Resolutions for Finite Groups," Annals Math. 94 (1960), 267-291. 